\title{
Effect of nitrogen availability on the poly-3-D-hydroxybutyrate accumulation by engineered Saccharomyces cerevisiae
}

Diogo J. Portugal-Nunes ${ }^{1}$, Sudhanshu S. Pawar², Gunnar Lidén² and Marie F. Gorwa-Grauslund ${ }^{1 *}$

\begin{abstract}
Poly-3-D-hydroxybutyrate (or PHB) is a polyester which can be used in the production of biodegradable plastics from renewable resources. It is naturally produced by several bacteria as a response to nutrient starvation in the excess of a carbon source. The yeast Saccharomyces cerevisiae could be an alternative production host as it offers good inhibitor tolerance towards weak acids and phenolic compounds and does not depolymerize the produced PHB. As nitrogen limitation is known to boost the accumulation of PHB in bacteria, the present study aimed at investigating the effect of nitrogen availability on PHB accumulation in two recombinant S. cerevisiae strains harboring different xylose consuming and PHB producing pathways: TMB4443 expressing an NADPH-dependent acetoacetyl-CoA reductase and a wild-type S. stipitis XR with preferential use of NADPH and TMB4425 which expresses an NADH-dependent acetoacetyl-CoA reductase and a mutated XR with a balanced affinity for NADPH/NADH. TMB4443 accumulated most PHB under aerobic conditions and with glucose as sole carbon source, whereas the highest PHB concentrations were obtained with TMB4425 under anaerobic conditions and xylose as carbon source. In both cases, the highest PHB contents were obtained with high availability of nitrogen. The major impact of nitrogen availability was observed in TMB4425, where a 2.7-fold increase in PHB content was obtained. In contrast to what was observed in natural PHBproducing bacteria, nitrogen deficiency did not improve PHB accumulation in S. cerevisiae. Instead the excess available carbon from xylose was shunted into glycogen, indicating a significant gluconeogenic activity on xylose.
\end{abstract}

Keywords: Poly-3-D-hydroxybutyrate, PHB, Bioplastic, Saccharomyces cerevisiae, Nitrogen limitation, Glycogen

\section{Introduction}

Poly-3-D-hydroxybutyrate (PHB) was the first discovered microbial produced alkanoate (Khanna and Srivastava 2005). PHB is one of the best candidates for production of fully biodegradable plastics from renewable resources as it possesses similar characteristics as the most common plastics used in industry-polyethylene and polypropylene (Khanna and Srivastava 2005; Wang and Lee 1997). PHBs are also thermoplastic and biocompatible, which opens the possibility for medical applications (Verlinden et al. 2007; Yoneyama et al. 2015).

\footnotetext{
*Correspondence: marie-francoise.gorwa-grauslund@tmb.lth.se ${ }^{1}$ Applied Microbiology, Department of Chemistry, Lund University, PO Box 124, 22100 Lund, Sweden

Full list of author information is available at the end of the article
}

These compounds are naturally accumulated as intracellular carbon granules and energy storage material in several bacteria, the so-called 'natural producers', such as Cupriavidus necator, Bacillus megaterium and several species belonging to the genus Pseudomonas (Trotsenko and Belova 2000; Verlinden et al. 2007). PHB production from the acetyl coenzyme A (acetyl-CoA) intermediate occurs in a three step process consisting of (a) an acetylCoA acetyltransferase, which catalyzes the combination of two acetyl-CoA to acetoacetyl-CoA (Peoples and Sinskey 1989b), (b) an acetoacetyl-CoA reductase, which reduces acetoacetyl-CoA to 3-D-hydroxybutyryl-CoA (Peoples and Sinskey 1989b) and (c) a PHB synthase, catalyzing the final polymerization step (Peoples and Sinskey 1989a). The accumulation of PHB is generally optimal under excess of carbon and limiting levels of nitrogen, phosphorous and/or oxygen (Verlinden et al. 2007). 
Although the natural producers are reported to accumulate more than 60\% PHB per cell biomass (Budde et al. 2011; Schlegel et al. 1961), growth is slow and the PHB recovery might be challenging (Suriyamongkol et al. 2007). Moreover, the presence of endogenous depolymerases that can utilize the accumulated PHB as carbon/energy, reduces the overall production (Jendrossek and Handrick 2002). The price of the raw materials-so far sugars of agricultural origin-and their transportation can be two major contributors for the final cost. So, despite the numerous advantages compared to the traditional oil-based plastics, commercial bio-based PHB production has not been reported yet. Sugars derived from lignocellulosic biomass or other sugar-rich waste streams are alternative carbon sources, which have environmental advantages in terms of a reduced net release of carbon dioxide in comparison to crop-derived sugars (Dornburg et al. 2008). Hydrolysates from lignocellulosic biomass are composed of a mixture of several monomeric sugars, which can be used as carbon sources by microorganisms (Olsson and Hahn-Hägerdal 1996). For instance, spent sulfite liquor (SSL) is a sugar-rich stream generated by the sulfite based pulp-mills (Inskeep et al. 1951) that has been extensively studied over the past decades as a potential underutilized source of sugars. The relative proportion of monosaccharides in the SSL will strongly depend on the feedstock, where softwood-such as spruce and pine-will give high proportions of hexoses such as mannose and glucose, whereas SSL from hardwoods-such as Eucalyptus-will contain predominantly xylose (Lawford and Rousseau 1993) [see also recent review from (Pereira et al. 2013)]. However, a significant challenge with lignocellulosic feedstock is the presence, after hydrolysis, of weak acids and phenolic compounds that act as microbial inhibitors (Olsson and Hahn-Hägerdal 1996; Palmqvist and Hahn-Hägerdal 2000; Taherzadeh et al. 1997).

As an alternative to natural hosts, PHB pathways from natural PHB producers have been introduced in organisms that grow fast, cannot re-use accumulated PHB as a carbon source or are able to resist inhibitors. The bacterium Escherichia coli (Fidler and Dennis 1992; Kalousek and Lubitz 1995), the yeast Saccharomyces cerevisiae (Breuer et al. 2002; Kocharin et al. 2012; Leaf et al. 1996; Sandström et al. 2015), insect cells of armyworm and plants like tobacco (Poirier et al. 1992; Suriyamongkol et al. 2007) are some of the prominent examples. When using lignocellulose-derived sugars as raw materials, $S$. cerevisiae is probably one of the most suitable candidate host due to its fermentation capacity, robustness and extensive characterization (Olsson and Hahn-Hägerdal 1993; Ostergaard et al. 2000). PHB production from glucose was first attempted in S. cerevisiae through the expression of the PHB synthase gene (Leaf et al. 1996) and further boosted by expressing the acetyl-CoA acetyltransferase and acetoacetyl-CoA reductase genes from C. necator (Breuer et al. 2002; Carlson and Srienc 2006). PHB accumulation was further improved by increasing the availability of the precursor acetyl-CoA and the pool of the co-factor nicotinamide adenine dinucleotide phosphate (NADPH) (Kocharin et al. 2012, 2013). More recently, $\mathrm{PHB}$ formation from xylose as the sole carbon source was obtained under aerobic conditions in an engineered S. cerevisiae strain (TMB4443) carrying the oxido-reductive xylose pathway from S. stipitis (Sandström et al. 2015). By expressing an acetoacetyl-CoA reductase dependent on the cofactor nicotinamide adenine dinucleotide (NADH) from Allochromatium vinosum in combination with an improved xylose pathway, it was shown possible to obtain anaerobic PHB production from xylose resulting in the highest $\mathrm{PHB}$ content per cell (14 wt \%) reported so far for S. cerevisiae in the strain TMB4425 (de Las Heras et al. 2016).

Redirection of carbon flux into a desired product by nutrient limitation is a well-established strategy in several industrial processes, e.g. the citric acid fermentation (Anastassiadis et al. 2002; Yalcin et al. 2010). Growth limitation, caused by e.g. limiting the availability of nitrogen, in the presence of a carbon source, is a key factor for obtaining high PHB titers in natural producers (Wang and Lee 1997). In non-engineered S. cerevisiae, nitrogen limitation causes accumulation of other storage carbohydrates, primarily glycogen, but to some extent also trehalose (Lillie and Pringle 1980). The accumulation of glycogen and trehalose is proportional to the duration of the $G_{1}$ phase of the growth cycle-interphase step where growth organelles and cell material are dividing but DNA replication is not taking place (Paalman et al. 2003). Within a given concentration range, glycogen confers a competitive advantage in growth over cells that do not accumulate this storage compound (Anderson and Tatchell 2001). As for trehalose, it is associated with stress protection, acting as a stabilizer of the different cell compartments under heat stress, for example Crowe and Chapman 1984; Hottiger et al. 1994). The accumulation of both carbohydrates is tightly regulated in yeast and confers survival advantages to the cell (François and Parrou 2001).

The recently described PHB production in S. cerevisiae from xylose together with the extensive on-going research on boosting the intracellular pool of the precursor acetyl-CoA and fine-tuning the redox state of the cell, opens up several routes for the optimization of the PHB formation. The aim of the present study was to specifically investigate the effect of nitrogen availability on the PHB accumulation of two strains of $S$. cerevisiae engineered for PHB production. Glucose or xylose were used 
as carbon sources, since these are likely to be present in various residue streams derived from lignocellulosic biomass. The two strains used, TMB4443 and TMB4425, were both able to consume xylose by an introduced twostep conversion of xylose to xylulose by xylose reductase (XR) and xylitol dehydrogenase (XDH) (de Las Heras et al. 2016; Sandström et al. 2015). However, the strains were based on expression of different heterologous xylose and acetoacetyl-CoA reductases, with different affinities for the co-factors NADH and NADPH (Fig. 1). Cultivation experiments were performed in media with different ratios between the carbon and the nitrogen source, and consumption of sugars as well as the accumulation of $\mathrm{PHB}$, glycogen and trehalose were measured. To the best of our knowledge, the effect of nitrogen deficiency on PHB accumulation and its relation with the other storage carbon compounds found in S. cerevisiae has not been investigated yet.

\section{Materials and methods}

Strains, media and culture conditions

Genetically engineered strains of $S$. cerevisiae, namely TMB4443 (Sandström et al. 2015) and TMB4425 (de Las Heras et al. 2016) with the ability to accumulate PHB were used in this study. The PHB producing pathway in TMB4443 consists of the genes PhaA, PhaB1 and Phac1 from $C$. necator and harbors the wild-type XR-XDH pathway from Scheffersomyces stipitis (Sandström et al. 2015E). In TMB4425, the acetoacetyl-CoA reductase that is coded by PhaB1 was replaced by an alternative NADH-dependent reductase from Allochromatium vinosum (de Las Heras et al. 2016). Another particular trait of TMB4425 is that harbors a mutated version of the XR instead of the wild-type, which confers a balance affinity for NADH and NADPH. TMB4444 was used as negative control since it lacks the PHB producing pathway (Sandström et al. 2015E). In all cases, the xylose consuming

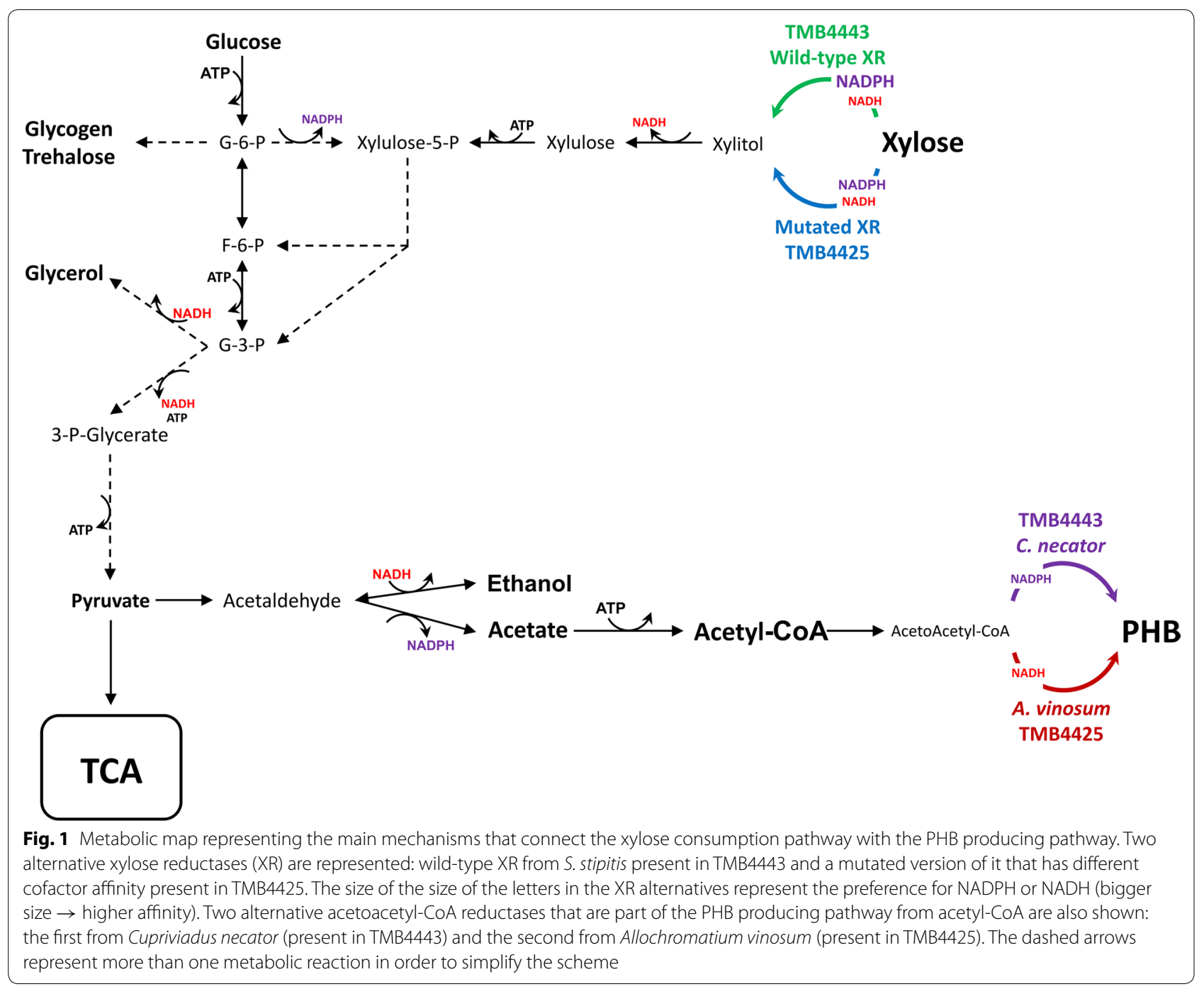


pathways were optimized by overexpressing the pentose phosphate pathway (de Las Heras et al. 2016; Sandström et al. 2015).

The pre-cultures were recovered by transferring a $10 \mu \mathrm{L}$ loop of cells from the glycerol stocks stored at $-80{ }^{\circ} \mathrm{C}$ into $5.0 \mathrm{~mL}$ of a growth medium containing $13.4 \mathrm{~g}$ yeast nitrogen base (YNB)/L, $50 \mathrm{mM}$ potassium hydrogen phthalate buffer at $\mathrm{pH} 5.5$ and was supplemented either with $50 \mathrm{~g}$ D-glucose/L or $50 \mathrm{~g} \mathrm{D}$-xylose/L, depending on the carbon source used in the further cultivations. Cells were grown in a $50 \mathrm{~mL}$ sterile conical centrifuge tube (Sarstedt AG \& Co., Nümbrecht, Germany) and incubated at $30^{\circ} \mathrm{C}$ and $180 \mathrm{rpm}$ until the end of the exponential growth phase was reached.

Two different Yeast Base (YB) media were tested in the further aerobic cultivations and anaerobic fermentations: (1) N-high, which contained 13.4 g YNB without amino acids and ammonium sulfate/L, $10.0 \mathrm{~g}$ ammonium sulfate $/ \mathrm{L}$, and $50 \mathrm{mM}$ potassium hydrogen phthalate buffer at pH 5.5 and (2) N-def, which contained $13.4 \mathrm{~g}$ YNB without amino acids and ammonium sulfate/L, $50 \mathrm{mM}$ potassium hydrogen phthalate buffer at $\mathrm{pH}$ 5.5. Depending on the experimental design the growth media $N$-high and $N$-def were supplemented with either $50 \mathrm{~g}$ glucose/L (termed as Glc-N-high and Glc-N-def, respectively) or $50 \mathrm{~g}$ xylose/L (termed as $\mathrm{Xyl}-\mathrm{N}-\mathrm{high}$ and $\mathrm{Xyl}-\mathrm{N}$-def, respectively). In the case of the anaerobic fermentations, $0.01 \mathrm{~g}$ ergosterol/L and $0.42 \mathrm{~g}$ Tween $80 / \mathrm{L}$ were added to the medium.

\section{Cultivations and fermentations \\ PHB accumulation tests under high nitrogen availability}

Yeast cell pellets obtained from the pre-cultures were centrifuged (4000 rpm, $5 \mathrm{~min}, 4{ }^{\circ} \mathrm{C}$ ), resuspended and washed with sterile saline solution $(0.9 \% \mathrm{NaCl})$. The cells were re-suspended in the cultivation medium and inoculated such that an initial optical density of 0.05 was obtained. The optical density was measured at a wavelength of $620 \mathrm{~nm}\left(\mathrm{OD}_{620 \mathrm{~nm}}\right)$ by using an Ultrospec 2100 Pro spectrophotometer (Amersham Biosciences Corp., New Jersey, USA). Yeast strains were cultivated in $250 \mathrm{~mL}$ shake-flasks with baffles containing $50 \mathrm{~mL}$ medium for the aerobic cultivations. A sterile foam plug was used in order to keep the aerobicity during the experiment. In the case of anaerobic fermentations, $250 \mathrm{~mL}$ shake-flasks without baffles but with a rubber stopper and a glycerol trap were used. Nitrogen gas $\left(\mathrm{N}_{2}\right)$ was continuously sparged with the aid of a sterile needle that was in contact with the liquid medium over the fermentation. The flasks were incubated in a shaking incubator operated at $30{ }^{\circ} \mathrm{C}$ and $180 \mathrm{rpm}$. TMB4443 was tested in Glc-Nhigh and $\mathrm{Xyl}$-N-high under aerobic conditions, and only in Glc-N-high under anaerobic conditions. TMB4425 was evaluated in Glc-N-high and Xyl-N-high both under aerobic and anaerobic conditions. $\mathrm{OD}_{620 \mathrm{~nm}}$ was followed overtime (Ultrospec 2100 Pro spectrophotometer, Amersham Biosciences, Corp., USA) and samples for cell dry weight (CDW), extracellular metabolites and PHB analysis were collected in technical duplicate. For each condition and strain evaluated, two biological replicates were performed.

\section{PHB accumulation tests under nitrogen deficiency}

Yeast cell pellets obtained from the pre-cultures were centrifuged ( $4000 \mathrm{rpm}, 5 \mathrm{~min}, 4{ }^{\circ} \mathrm{C}$ ), re-suspended and washed with $0.9 \% \mathrm{NaCl}$. An intermediate step for cell proliferation was made by growing the cells in $50 \mathrm{~mL}$ of either Glc-N-high or Xyl-N-high by inoculating with the washed cells such that initial $\mathrm{OD}_{620 \mathrm{~nm}}$ corresponding to 0.05 was achieved. The cultures were incubated at $30{ }^{\circ} \mathrm{C}$ and were subjected to shaking at $180 \mathrm{rpm}$. The cells were harvested by centrifugation at the end of the exponential growth phase and were washed and re-suspended subsequently. These re-suspended cells were inoculated at an initial $\mathrm{OD}_{620 \mathrm{~nm}}$ of 12.5 , corresponding to approximately $4.0 \mathrm{~g}$ biomass/L. TMB4443 was cultivated aerobically in $250 \mathrm{~mL}$ shake-flasks with baffles containing $50 \mathrm{~mL}$ medium $(G l c-N-d e f)$. A sterile foam plug was used to in order to keep the aerobicity during the experiment. In the case of anaerobic fermentations, TMB4425 was evaluated in $250 \mathrm{~mL}$ shake-flasks without baffles containing $50 \mathrm{~mL}$ of $\mathrm{Xyl}-\mathrm{N}$-def medium. The shake-flasks were equipped with a rubber stopper and a glycerol trap and $\mathrm{N}_{2}$ was continuously sparged with the aid of a sterile needle that was in contact with the liquid medium over the fermentation. For each condition and strain evaluated, two biological replicates were performed.

\section{Analytical assays \\ Biomass}

Biomass was quantified by measuring CDW. CDW corresponding to the end-point of the cultivations was measured in duplicate by filtering a measured volume of the culture through a pre-weighed $0.45 \mu \mathrm{m}$ pore size nitrocellulose filter (PALL, Michigan, USA). After the drying process the filters were equilibrated to room temperature in a desiccator and then weighed. Biomass was correlated to $\mathrm{OD}_{620 \mathrm{~nm}}$ by a single-point calibration, based on the final time point measurement.

\section{Extracellular metabolites and PHB}

Concentrations of glucose, xylose, xylitol, glycerol, acetate and ethanol were determined by high-performance liquid chromatography (HPLC, Waters, Massachusetts, USA). The compounds were separated using an Aminex HPX-87H ion exchange column (Bio-Rad, California, 
USA) preceded by a Micro-Guard Cation- $\mathrm{H}$ guard column (Bio-Rad, California, USA). Separation was performed at $45{ }^{\circ} \mathrm{C}$ with $5 \mathrm{mM} \mathrm{H}_{2} \mathrm{SO}_{4}$ as mobile phase at a flow rate of $0.6 \mathrm{~mL} / \mathrm{min}$. All compounds were quantified by refractive index detection (Waters, Massachusetts, USA). A seven-point calibration was made for each compound to determine the concentrations and each sample was analyzed at least in technical duplicate.

The quantification of PHB was based on the method described by Law and Slepecky, where the conversion of the polymer into crotonic acid is catalyzed by hot concentrated sulfuric acid (Law and Slepecky 1961). The extraction and analytical methods were followed as described Sandström et al. (2015).

\section{Glycogen and trehalose}

Glycogen and trehalose extraction was done simultaneously for each collected sample by using the method described by Parrou and François (1997) that is based on the alkaline digestion of yeast cells in $0.25 \mathrm{M} \mathrm{Na}_{2} \mathrm{CO}_{3}$ at $95^{\circ} \mathrm{C}$ (Becker 1978).

Glycogen concentrations were determined using the Glycogen Assay Kit (\#MAK016, Sigma-Aldrich, Missouri, USA). The samples analyzed corresponded to the initial $(0 \mathrm{~h})$ and end-points $(170 \mathrm{~h})$ of the experiments performed under nitrogen deficiency with the strains TMB4425 (Xyl-N-def), TMB4443 (Glc-N-def and Xyl-Ndef) and TMB4444 (Xyl-N-def). The end-point samples corresponding to the assays performed in $\mathrm{Xyl}$-N-high with TMB4425 and Glc-N-high with TMB4443 were also analyzed. Samples were diluted 50-100 times and analyzed in technical duplicates. The glycogen concentration was calculated from a six-point calibration curve.

Trehalose was determined by ultra-performance liquid chromatography (UPLC) (Waters, Massachusetts, USA). Separation was performed with the aid of a Waters UPLC BEH Amide $2.1 \times 100 \mathrm{~mm}$ (Waters, Massachusetts, USA) column at $50{ }^{\circ} \mathrm{C}$ and $0.4 \mathrm{~mL} \mathrm{~min}{ }^{-1}$. A gradient of concentrations of acetonitrile and aqueous solution of ammonium acetate was created according to the method recently described by Almqvist et al. (2016). Trehalose was quantified by evaporative light scattering detection (Waters, Massachusetts, USA).

\section{Results}

\section{Effect of nitrogen availability on PHB production using glucose as sole C-source}

The performance of S. cerevisiae TMB4443, having NADPH preference for xylose and PHB pathways, was evaluated under aerobic conditions to compare the effect of the absence or excess of nitrogen. As initial work indicated that TMB4443 accumulated higher intracellular concentrations of $\mathrm{PHB}$ when glucose was used instead of xylose (data not shown), glucose was chosen as the principle carbon source for TMB4443 in the assessment of effects of nitrogen availability.

TMB4443 was able to consume all of the added glucose even in the absence of ammonium sulfate (Glc$\mathrm{N}$-def, Fig. 2a). However, upon addition of ammonium sulfate (Glc-N-high, Fig. 2c), the cells consumed glucose at a rate that was at least twofold higher, as glucose was depleted after $50 \mathrm{~h}$ of cultivation compared to approximately $125 \mathrm{~h}$ required by the cells in cultures lacking ammonium sulfate (Glc-N-def, Fig. 2a). In the case of Glc-N-high, where a low starting biomass of $0.01 \mathrm{~g}$ $\mathrm{CDW} / \mathrm{L}$ was used, $4.8 \pm 0.4 \mathrm{~g} \mathrm{CDW} / \mathrm{L}$ was formed (Fig. 2d), indicating that the cell growth was not limited by the nitrogen source or other nutrients. The same intended initial biomass was tried in the Glc-Ndef experiments but no significant growth was observed (data not shown). For this reason, high initial cell density $(3.8 \mathrm{~g} \mathrm{CDW} / \mathrm{L})$ was used for the $\mathrm{N}$-def experiments. Initially, glucose was rapidly converted mainly into ethanol which reached its maximum concentration after $50 \mathrm{~h}$ of cultivation. Ethanol production under aerobic conditions with high residual glucose concentration was expected since $S$. cerevisiae is a Crabtree-positive yeast (De Deken 1966). At this time point, where the titer of $\mathrm{PHB}$ was $0.12 \mathrm{~g} \mathrm{PHB} / \mathrm{L}$, PHB accumulation stopped. Subsequent ethanol consumption did not lead to further PHB accumulation (Fig. 2a). In Glc-N-high conditions, the analysed metabolites reached higher titers. The maximum glycerol and ethanol titers were observed after $50 \mathrm{~h}$ of cultivation. The maximum acetate titer, achieved within $70 \mathrm{~h}$, was about sixfold higher than that achieved with Glc-N-def assay. The final PHB titer was $0.11 \mathrm{~g} \mathrm{PHB} / \mathrm{L}$ and since the initial biomass concentration was low, no PHB was detected at the beginning of the cultivations (Fig. 2c).

Although similar final PHB titers were obtained at the end of both experiments, a substantial fraction of the PHB was present in the biomass already at the start in the case of nitrogen limitation because the cells were pre-grown in a medium containing glucose with excess of ammonium sulfate - condition that favors PHB accumulation (Fig. 2c). The net PHB production $\left(\mathrm{PHB}_{\text {final concentration }}-\mathrm{PHB}_{\text {initial concentration }}\right)$ was only $0.05 \mathrm{~g} \mathrm{PHB} / \mathrm{L}$ during the consumption of the glucose under nitrogen deficient conditions and it should be compared to $0.11 \mathrm{~g} \mathrm{PHB} / \mathrm{L}$ obtained in the presence of ammonium sulfate (Fig. 3).This was accompanied by a higher PHB content (\% PHB/CDW) when ammonium sulfate was supplied (Fig. 3; Table 1). 


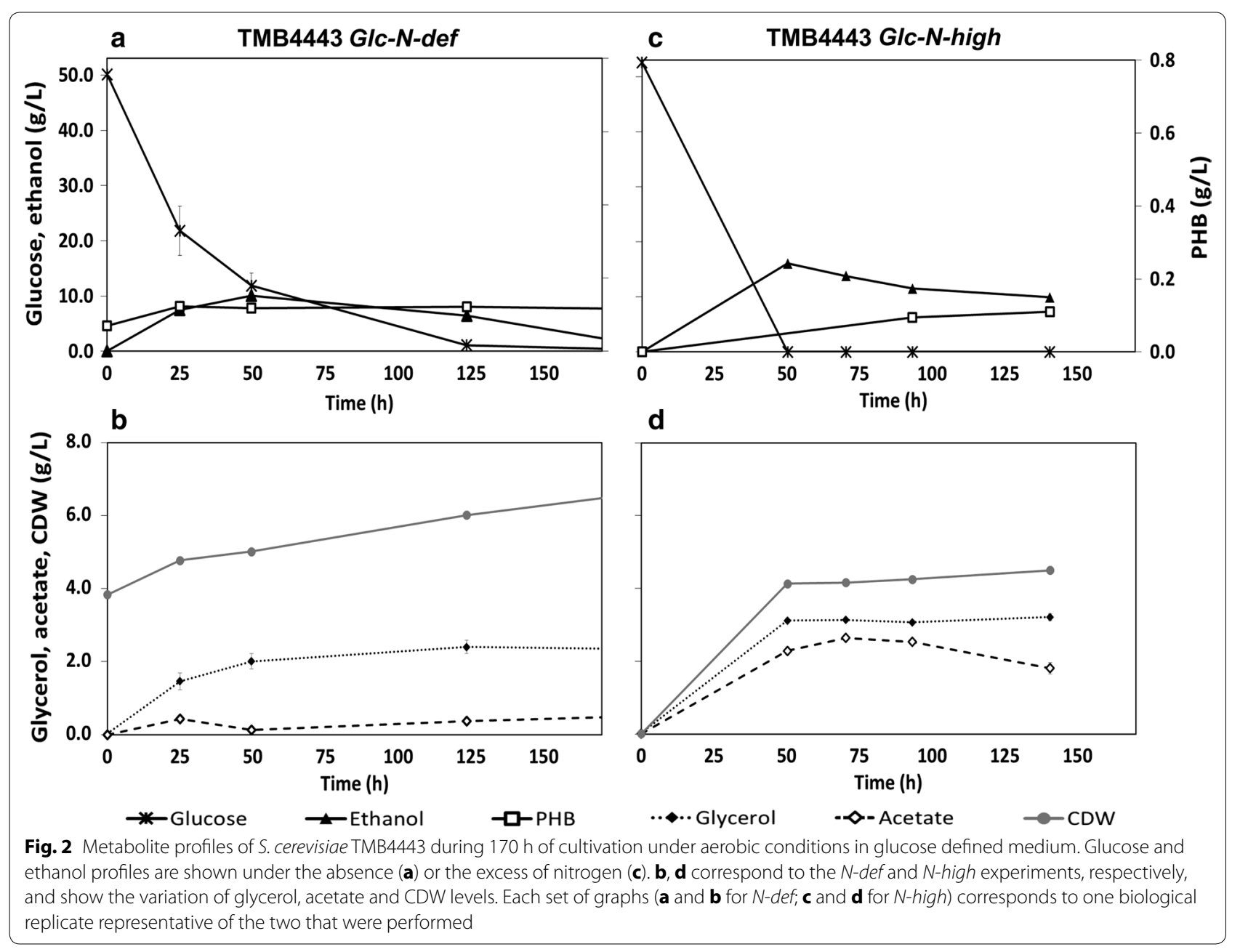

\section{Effect of nitrogen availability on PHB production using xylose as sole C-source}

The effect of nitrogen availability on PHB accumulation was also evaluated in S. cerevisiae TMB4425, which has a preference for $\mathrm{NADH}$ as a reducing co-factor in both the added xylose and PHB pathways. Similar to TMB4443, TMB4425 was also characterized in a medium containing either glucose or xylose and under aerobic or anaerobic conditions. As TMB4425 accumulated more PHB when grown on xylose under anaerobic conditions (data not shown), only anaerobic conditions were applied while evaluating the effect of nitrogen availability on the PHB accumulation by TMB4425 (Fig. 4).

As established earlier a high starting cell density was needed in the $N$-def experiments which allowed xylose to be depleted in $X y l-N$-def (Fig. 4a). The cells accumulated PHB during growth on xylose, reaching a final and maximum PHB titer of $0.43 \pm 0.04 \mathrm{~g} \mathrm{PHB} / \mathrm{L}$. In contrast

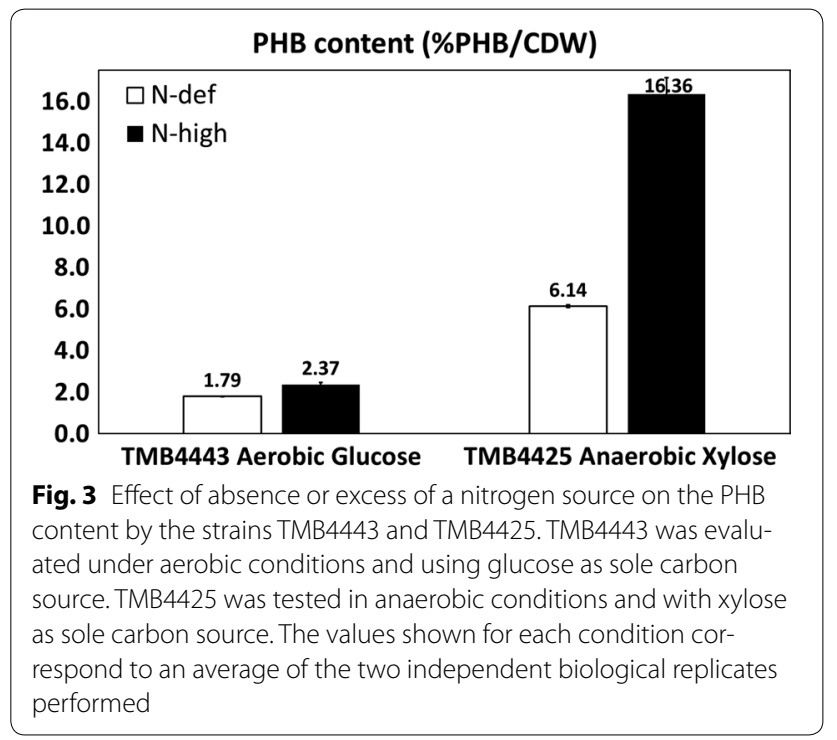




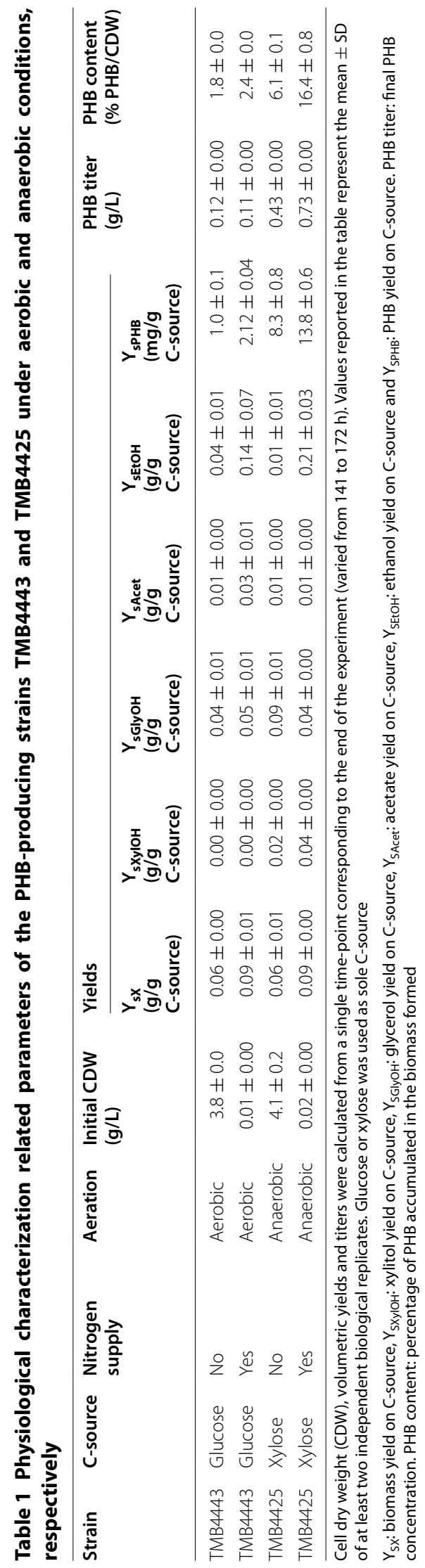




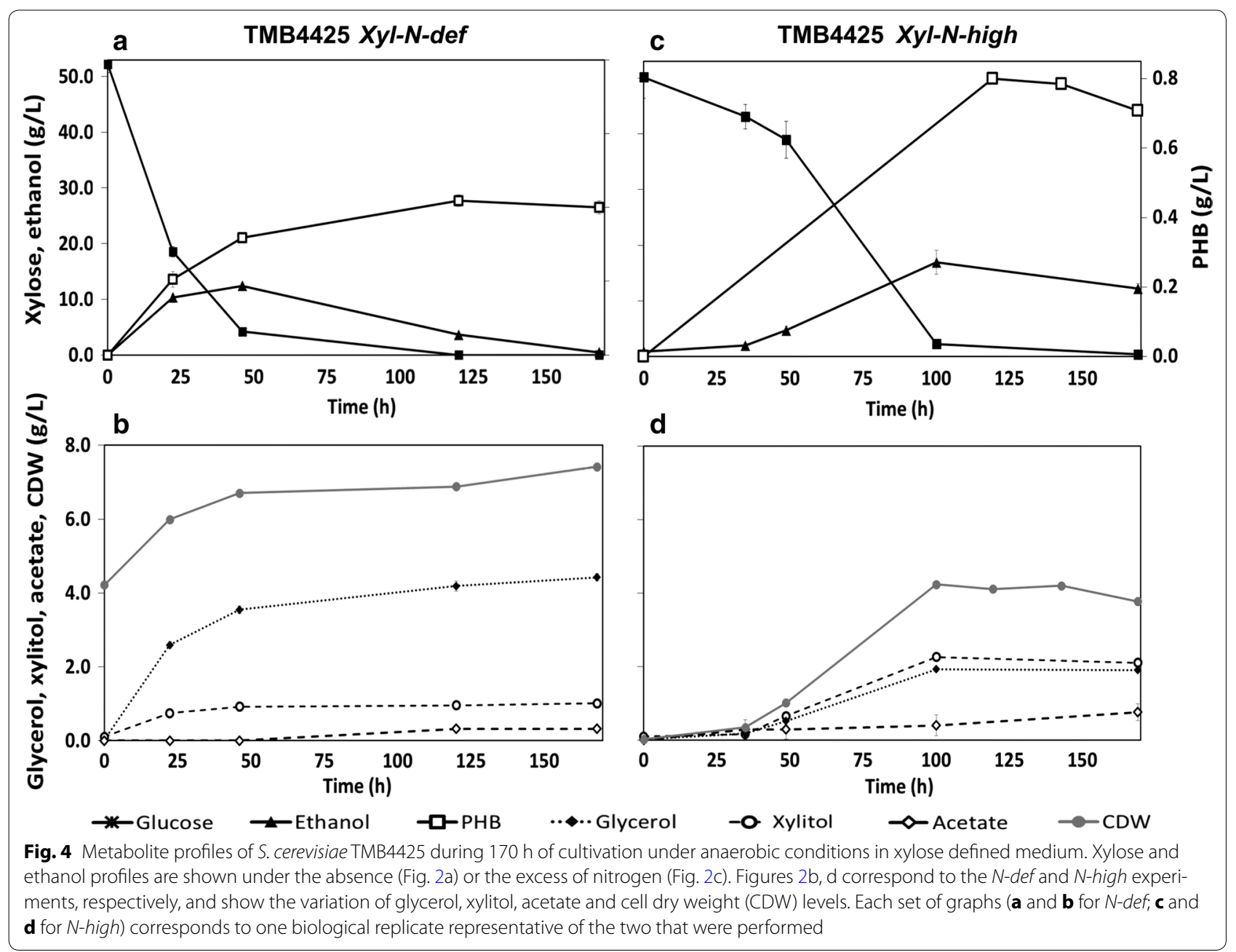

to the Glc-N-def experiment, no PHB was detected at the beginning of $X y l-N$-def. This was due to the fact that cells were pre-grown in conditions unfavorable to PHB accumulation i.e. on xylose in aerobic conditions. The initial xylose consumption resulted mostly in biomass and ethanol formation, but also in low amounts of glycerol and xylitol. At $50 \mathrm{~h}$, ethanol titer peaked after which it started declining. Ethanol consumption under anaerobic conditions is not feasible in S. cerevisiae due to a redox imbalance and low availability of ATP (Henningsen et al. 2015), but the decrease was instead due to evaporation resulting from the continuous sparging with nitrogen gas. Biomass formation and glycerol production continued until the end of the experiment (Fig. 4b).

When ammonium sulfate was added, $X y l-N$-high, a low initial biomass inoculum was used which gave a lower volumetric sugar uptake rate than in the previous case (Fig. 4c). However, xylose uptake rate increased and xylose was nearly depleted after $100 \mathrm{~h}$ of fermentation.
Xylose consumption resulted mainly in biomass and ethanol formation (Fig. 4). The presence of a nitrogen source resulted in higher xylitol than glycerol accumulation (Fig. 4d), in contrast to what was observed in $X y l-N$-def (Fig. 4b). The maximum PHB titer was achieved after $120 \mathrm{~h}(0.81 \pm 0.01 \mathrm{~g} \mathrm{PHB} / \mathrm{L})$, and then it slightly decreased until the end of the fermentation. Since PHB accumulates intracellularly, any cell lysis might release PHB in the medium, which will not be accounted for in the PHB extraction assay that relies on the analysis of the cell pellet. Thus, it is possible that cell lysis caused an underestimation of the PHB levels at the end-point.

The addition of ammonium sulfate thus resulted in a more efficient $\mathrm{PHB}$ production, giving a higher final PHB concentration. Importantly, the intracellular PHB concentration (\% $\mathrm{PHB} / \mathrm{CDW})$ was 2.7 -fold higher in the medium containing nitrogen than in the nitrogen deficient medium (Fig. 3; Table 1). 


\section{Intracellular concentrations of glycogen, trehalose and PHB}

Glycogen, $\mathrm{PHB}$ and trehalose were determined for TMB4425 and TMB4443. As a control, strain TMB4444 that lacks the PHB producing pathway was also evaluated.

The initial samples $(0 \mathrm{~h})$ were taken from the experiments performed with nitrogen deficient media $(N-d e f)$, since accurate quantification of glycogen, trehalose and PHB required a sufficient amount of biomass (approximately $10^{6}$ cells). Therefore the initial samples reflect the intracellular accumulation of the pre-culture, i.e. a medium with high nitrogen content.

In the control strain TMB4444, only glycogen was detected as carbon storage compound (data not shown), whereas PHB and glycogen were found in TMB4425 and TMB4443. In those strains, the distribution of the intracellular concentrations of the three storage molecules was compared (Fig. 5). The inoculum pre-culture used for the $N$-def experiments had a very low glycogen content and $97-98 \%$ of the carbon storage was PHB. During consumption of sugars in the absence of a nitrogen source, there was an increase in the glycogen accumulation, reaching $0.25 \pm 0.00$ and $0.78 \pm 0.07$ g glycogen $/ \mathrm{L}$ in glucose and xylose, respectively. After normalization, these glycogen levels correspond to 62 and $93 \%$ of the final total storage carbon, respectively. In contrast, only PHB was found as storage compound in the experiments in which both ammonium sulfate and glucose were supplied. For strain TMB4425, 98\% of the storage polymers was PHB under anaerobic conditions with xylose as carbon source and in the presence of a nitrogen source after $170 \mathrm{~h}$ (Fig. 5). The pre-culture of TMB4425 did not show $\mathrm{PHB}$ accumulation (i.e. the value at $0 \mathrm{~h}$ ), i.e. there was no polymer accumulated when cells were pre-grown aerobically prior to inoculation into their respective nitrogen deficient media. This result correlates well with metabolite profiles shown in Fig. 4a.

The absence of ammonium sulfate during $170 \mathrm{~h}$ induced glycogen production in TMB4443 (Glc-N-def) and TMB4425 (Xyl-N-def), suggesting that this effect was not strain or substrate dependent. In order to confirm this hypothesis, TMB4443 was also evaluated in xylose media under aerobic conditions to determine if a different carbon source would result in a different pattern. In this case, after $170 \mathrm{~h}$ more than $90 \%$ of the total carbon storage was glycogen (data not shown).

\section{Discussion}

The intracellular PHB levels reported here are clearly lower than the intracellular levels obtained by some natural prokaryotic $\mathrm{PHB}$ producers (PHB content above 50\%). However, those levels were not obtained using xylose as a carbon source. Furthermore, the robustness and inhibitor tolerance of $S$. cerevisiae is an advantage for utilization of xylose-rich waste streams, e.g. from the paper industry. In the present study, we show that the removal of nitrogen from the culture medium has a negative impact on the PHB content in S. cerevisiae. In previous studies where a decreased amount of nitrogen in the medium did not improve PHB accumulation in S. cerevisiae (Carlson and Srienc 2006) or in engineered Komagataella pastoris (formerly known as Pichia pastoris) (Vijayasankaran et al. 2005), it was speculated that the low nitrogen level would affect the level of recombinant protein production (Carlson and Srienc 2006). Here we observe that, under nitrogen starvation, $\mathrm{PHB}$ production also directly competes

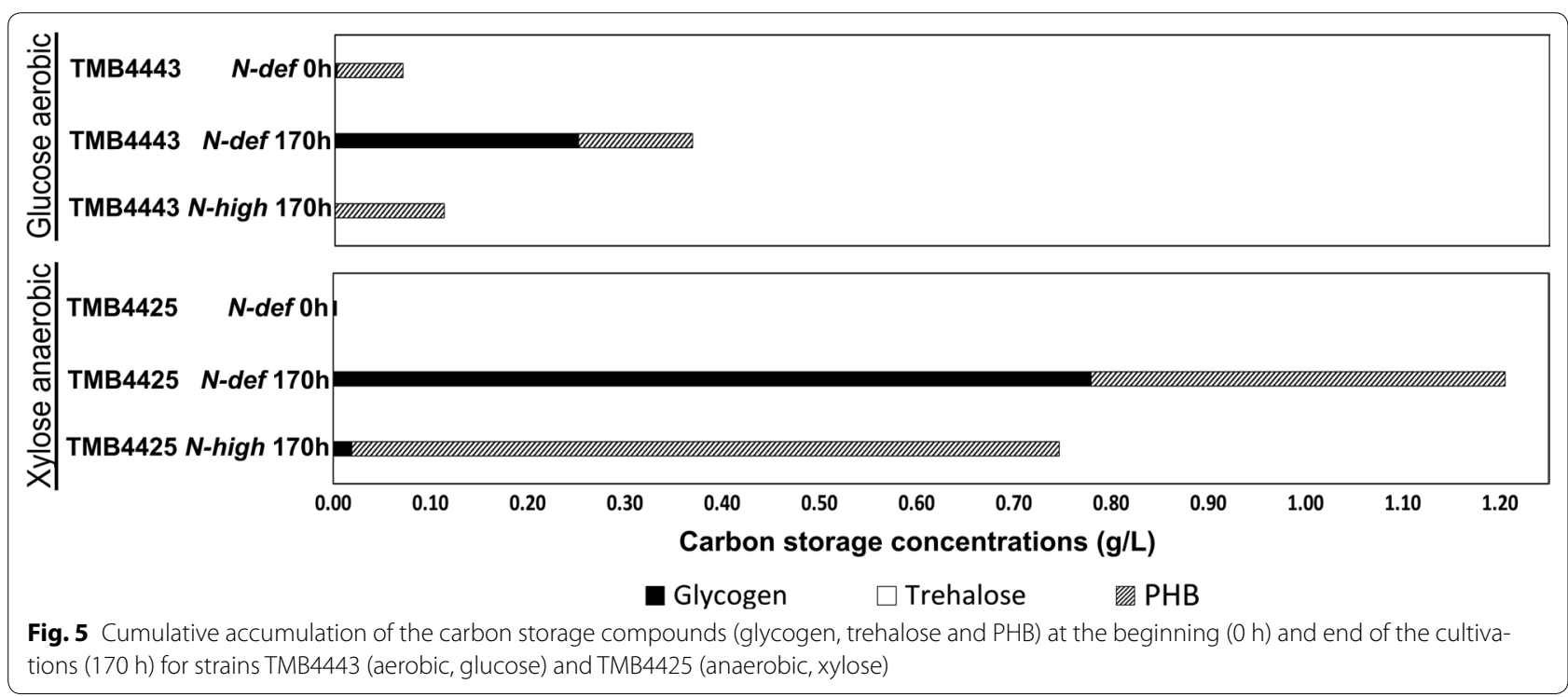


for carbon with glycogen formation and that this effect is independent of the engineering strategy, aeration and carbon source.

The availability of glucose-6-phosphate (G6P) that diverts directly from glucose, is considered to be the key for the regulation of glycogen and trehalose accumulation in S. cerevisiae (François and Parrou 2001). Here glycogen accumulation in the absence of nitrogen is observed not only on glucose but also on xylose, with both strains and under aerobic or anaerobic conditions. This suggests that at least one of the glycolytic intermediates that are formed from xylose conversion into central metabolism, namely fructose-6-phosphate (F6P) and/or glyceraldehyde3-phosphate (G3P) (Fig. 1), are channeled upwards to G6P via gluconeogenic enzymes including the enzyme phosphoglucose isomerase (PGI) that interconverts G6P and F6P. This is in agreement with transcriptomics data indicating increased transcription of genes involved on gluconeogenesis on xylose (Jin et al. 2004; Runquist et al. 2009). A decrease in PGI activity has previously been shown to negatively impact xylose uptake (Jeppsson et al. 2002) and it was then speculated that the decreased NADPH, arising from low G6P being channeled to the oxidative branch of the pentose phosphate pathway (PPP) (Fig. 1), directly affected the NADPH-dependent xylose reduction to xylitol by xylose reductase (XR). Here we observe glycogen formation under nitrogen starvation and also for a strain carrying a XR with a balanced cofactor preference for NADH and NADPH, which implies that the gluconeogenic flux is probably not regulated by the NADPH need of the XR enzyme nor by the NADPH need for biomass synthesis. Instead it might constitute an overflow resulting from the deregulation of gluconeogenic enzymes. However, this carbon redirection will result in less ATP being generated per xylose. Reduced ATP and enhanced G6P will promote glycogen synthesis since G6P is a stimulator and ATP an inhibitor of the glycogen synthase (François et al. 1988).

In contrast to previous reports with recombinant PHB producing S. cerevisiae (Kocharin et al. 2012; Vijayasankaran et al. 2005), in the present study PHB production was observed during the growth phase on both glucose and xylose. In the evaluated strain, the PHB genes were expressed under constitutive promoters, that is without any regulation, as opposed to what happens in natural producers (Pötter et al. 2002). The presence of PHB enzymes under growing conditions together with the availability of precursors such as acetyl-CoA and $\operatorname{NAD}(\mathrm{P}) \mathrm{H}$, which is favored by the high levels of nitrogen, explains the PHB accumulation during growth. Still, PHB competes with biomass synthesis for these precursors, so the level of PHB accumulation is restricted. In the bacteria that naturally accumulate $\mathrm{PHB}$, this polymer can be re-utilized as a carbon source in starvation periods.
However, as yeast does not contain enzymes necessary for degradation of PHB, PHB accumulates as a true "byproduct" of the metabolism since the cells do not directly benefit from its accumulation.

Notwithstanding the differences in the fermentation conditions, the PHB pathway present in the strain TMB4425 was demonstrated to be more efficient for PHB production in yeast than the one present in TMB4443. This result is in agreement with the recent observations of Muñoz de las Heras and co-workers (2016), where it was shown that the replacement of the acetoacetylCoA reductase $(P h a B)$ from $C$. necator by the NADHdependent reductase from the anoxygenic purple sulfur bacteria A. vinosum, resulted in higher PHB content and titer under oxygen-limited conditions (de Las Heras et al. 2016). Anaerobic bioprocesses are usually associated with certain advantages for industrial applications due to the lower operating costs for aeration than those required for fully aerobic processes (Kozak et al. 2014; Weusthuis et al. 2011). Under anaerobic conditions, $\mathrm{NADH}$ is generated in the glycolysis of S. cerevisiae and as a result of anabolic reactions. Reoxidation to $\mathrm{NAD}^{+}$is obtained through reduction of acetaldehyde to ethanol as well as by reduction of dihydroxyacetone phosphate to glycerol-3-phosphate (which is subsequently dephosphorylated to glycerol) in wild-type strains. In the strain holding the NADH-coupled acetoacetyl-CoA reductase, the PHB pathway can also serve to reoxidize NADH and maintain the co-factor balance in the cell. The decreased need for NADPH in the PHB pathway of TMB4425, may also be beneficial since it does not compete for NADPH needed for biosynthetic purposes.

In conclusion, the current study demonstrates that PHB accumulation in the evaluated $S$. cerevisiae strains with heterologous PHB formation pathways was higher in the presence of a nitrogen source in the medium. The attempt to redirect the carbon flux towards PHB by limiting cell growth clearly competes with the tightly regulated natural mechanism of carbon storage of S. cerevisiae under nitrogen limitation. Interestingly, glycogen accumulation from xylose was detected, indicating a significant gluconeogenic activity on this pentose. The NADH-coupled PHB producing pathway, in combination with a mutated XR in the strain TMB4425, was the most efficient pathway for PHB production from xylose, resulting in an intracellular PHB content as high as $16 \%$.

\section{Abbreviations}

Acetyl-CoA: acetyl coenzyme A; CDW: cell dry weight; F6P: fructose-6-phosphate; Glc: glucose; G3P: glyceraldehyde-3-phosphate; G6P: glucose-6-phosphate; HPLC: high-performance liquid chromatography; NAD ${ }^{+} / \mathrm{H}$ : nicotinamide adenine dinucleotide; $\mathrm{NADP}^{+} / \mathrm{H}$ : nicotinamide adenine dinucleotide phosphate; N-high: nitrogen excess medium; N-def: nitrogen deficient

medium; PGI: phosphoglucose isomerase; PHA: polyhydroxyalkanoate; PHB: 
polyhydroxybutyrate; PPP: pentose phosphate pathway; SSL: spent sulfite liquor; wt: wild-type; XDH: xylitol dehydrogenase; XK: xylulokinase; XR: $x y l o s e$ reductase; Xyl: xylose.

\section{Authors' contributions}

DPN and SP designed and coordinated the study, carrying out the experimental work. DPN drafted the manuscript. SP highly contributed for the manuscript revision and submission. GL and MFG participated in design of the study and helped drafting and reviewing the manuscript. All authors read and approved the final manuscript.

\section{Author details}

${ }^{1}$ Applied Microbiology, Department of Chemistry, Lund University, PO Box 124, 22100 Lund, Sweden. ${ }^{2}$ Department of Chemical Engineering, Lund University, PO Box 124, 22100 Lund, Sweden.

\section{Acknowledgements}

We would like to thank Alejandro Muñoz de Las Heras for providing the strains TMB4443 and TMB4425 and for valuable input. Henrik Almqvist is acknowledged for the development of the trehalose analytical method and for valuable input. Anders Sandström as well as our collaborators at SilicoLife and Biotrend SA are acknowledged for actively participating in discussion sessions.

\section{Competing interests}

The authors declare that they have no competing interests.

\section{Availability of data and materials}

The datasets supporting the conclusions of this article are included within the article.

\section{Funding}

The EU Commission (FP7 project BRIGIT, Contract nr 311935) is acknowledged for financial support.

Received: 27 January 2017 Accepted: 30 January 2017

Published online: 07 February 2017

\section{References}

Almqvist H, Sandahl M, Lidén G (2016) A rapid method for analysis of fermentatively produced d-xylonate using Ultra-High Performance Liquid Chromatography and Evaporative Light Scattering Detection. Biosci Biotech Biochem (Accepted)

Anastassiadis S, Aivasidis A, Wandrey C (2002) Citric acid production by Candida strains under intracellular nitrogen limitation. Appl Microbiol Biotechnol 60:81-87

Anderson C, Tatchell K (2001) Hyperactive glycogen synthase mutants of Saccharomyces cerevisiae Suppress the glc7-1 protein phosphatase mutant. J Bacteriol 183:821-829

Becker JU (1978) A method for glycogen determination in whole yeast cells. Anal Biochem 86:56-64

Breuer U, Terentiev Y, Kunze G, Babel W (2002) Yeasts as producers of polyhydroxyalkanoates: genetic engineering of Saccharomyces cerevisiae. Macromol Biosci 2:380-386

Budde CF, Riedel SL, Hübner F, Risch S, Popović MK, Rha C, Sinskey AJ (2011) Growth and polyhydroxybutyrate production by Ralstonia eutropha in emulsified plant oil medium. Appl Microbiol Biotechnol 89:1611-1619

Carlson R, Srienc F (2006) Effects of recombinant precursor pathway variations on poly [(R)-3-hydroxybutyrate] synthesis in Saccharomyces cerevisiae. J Biotechnol 124:561-573

Crowe JH, Crowe LM, Chapman D (1984) Preservation of membranes in anhydrobiotic organisms: the role of trehalose. Science 223(4637):701-703

De Deken R (1966) The Crabtree effect: a regulatory system in yeast. Microbiology 44:149-156

de Las Heras AM, Portugal-Nunes D, Rizza N, Sandström AG, Gorwa-Grauslund MF (2016) Anaerobic poly-3-d-hydroxybutyrate production from xylose in recombinant Saccharomyces cerevisiae using a NADH-dependent acetoacetyl-CoA reductase. Microbial Cell Fact 15:197
Dornburg V, Hermann BG, Patel MK (2008) Scenario projections for future market potentials of biobased bulk chemicals. Environ Sci Technol 42:2261-2267

Fidler S, Dennis D (1992) Polyhydroxyalkanoate production in recombinant Escherichia coli. FEMS Microbiol Rev 9:231-235

François J, Parrou JL (2001) Reserve carbohydrates metabolism in the yeast Saccharomyces cerevisiae. FEMS Microbiol Rev 25:125-145. doi:10.1111/j.1574-6976.2001.tb00574.x

François J, Villanueva ME, Hers HG (1988) The control of glycogen metabolism in yeast. Eur J Biochem 174:551-559

Henningsen BM, Hon S, Covalla SF, Sonu C, Argyros DA, Barrett TF, Wiswall E, Froehlich AC, Zelle RM (2015) Increasing anaerobic acetate consumption and ethanol yields in Saccharomyces cerevisiae with NADPH-specific alcohol dehydrogenase. Appl Environ Microbiol 81:8108-8117

Hottiger T, Virgilio C, Hall MN, Boller T, Wiemken A (1994) The role of trehalose synthesis for the acquisition of thermotolerance in yeast. Eur J Biochem 219:187-193

Inskeep GC, Wiley A, Holderby J, Hughes L (1951) Food yeast from sulfite liquor. Ind Eng Chem 43:1702-1711

Jendrossek D, Handrick R (2002) Microbial degradation of Polyhydroxyalkanoates. Annu Rev Microbiol 56:403-432

Jeppsson M, Johansson B, Hahn-Hägerdal B, Gorwa-Grauslund MF (2002) Reduced oxidative pentose phosphate pathway flux in recombinant xylose-utilizing Saccharomyces cerevisiae strains improves the ethanol yield from xylose. Appl Environ Microbiol 68:1604-1609

Jin Y-S, Laplaza JM, Jeffries TW (2004) Saccharomyces cerevisiae engineered for xylose metabolism exhibits a respiratory response. Appl Environ Microbiol 70:6816-6825

Kalousek S, Lubitz W (1995) High-level poly ( $\beta$-hydroxybutyrate) production in recombinant Escherichia coli in sugar-free, complex medium. Can J Microbiol 41:216-221

Khanna S, Srivastava AK (2005) Recent advances in microbial polyhydroxyalkanoates. Process Biochem 40:607-619. doi:10.1016/j. procbio.2004.01.053

Kocharin K, Chen Y, Siewers V, Nielsen J (2012) Engineering of acetyl-CoA metabolism for the improved production of polyhydroxybutyrate in Saccharomyces cerevisiae. AMB Expr 2:52

Kocharin K, Siewers V, Nielsen J (2013) Improved polyhydroxybutyrate production by Saccharomyces cerevisiae through the use of the phosphoketolase pathway. Biotechnol Bioeng 110:2216-2224

Kozak BU, van Rossum HM, Benjamin KR, Wu L, Daran J-MG, Pronk JT, van Maris AJ (2014) Replacement of the Saccharomyces cerevisiae acetylCoA synthetases by alternative pathways for cytosolic acetyl-CoA synthesis. Metab Eng 21:46-59

Law JH, Slepecky RA (1961) Assay of poly-beta-hydroxybutyric acid. J Bacteriol 82:33-36

Lawford HG, Rousseau JD (1993) Production of ethanol from pulp mill hardwood and softwood spent sulfite liquors by genetically engineered $E$. coli. Appl Biochem Biotechnol 39:667-685

Leaf TA, Peterson MS, Stoup SK, Somers D, Srienc F (1996) Saccharomyces cerevisiae expressing bacterial polyhydroxybutyrate synthase produces poly-3-hydroxybutyrate. Microbiology 142:1169-1180

Lillie SH, Pringle JR (1980) Reserve carbohydrate metabolism in Saccharomyces cerevisiae: responses to nutrient limitation. J Bacteriol 143:1384-1394

Olsson L, Hahn-Hägerdal B (1993) Fermentative performance of bacteria and yeasts in lignocellulose hydrolysates. Process Biochem 28:249-257

Olsson L, Hahn-Hägerdal B (1996) Fermentation of lignocellulosic hydrolysates for ethanol production. Enzyme Microb Technol 18:312-331

Ostergaard S, Olsson L, Nielsen J (2000) Metabolic engineering of Saccharomyces cerevisiae. Microbiol Mol Biol Rev 64:34-50

Paalman JWG, Verwaal R, Slofstra SH, Verkleij AJ, Boonstra J, Verrips CT (2003) Trehalose and glycogen accumulation is related to the duration of the G1 phase of Saccharomyces cerevisiae. FEMS Yeast Res 3:261-268. doi:10.1111/j.1567-1364.2003.tb00168.x

Palmqvist E, Hahn-Hägerdal B (2000) Fermentation of lignocellulosic hydrolysates. II: inhibitors and mechanisms of inhibition. Bioresour Technol 74:25-33

Parrou JL, François J (1997) A simplified procedure for a rapid and reliable assay of both glycogen and trehalose in whole yeast cells. Anal Biochem 248:186-188 
Peoples OP, Sinskey AJ (1989a) Poly-beta-hydroxybutyrate (PHB) biosynthesis in Alcaligenes eutrophus H16. Identification and characterization of the PHB polymerase gene (phbC). J Biol Chem 264:15298-15303

Peoples OP, Sinskey AJ (1989b) Poly-beta-hydroxybutyrate biosynthesis in Alcaligenes eutrophus H16. Characterization of the genes encoding beta-ketothiolase and acetoacetyl-CoA reductase. J Biol Chem 264:15293-15297

Pereira SR, Portugal-Nunes DJ, Evtuguin DV, Serafim LS, Xavier AM (2013) Advances in ethanol production from hardwood spent sulphite liquors. Process Biochem 48:272-282

Poirier Y, Dennis DE, Klomparens K, Somerville C (1992) Polyhydroxybutyrate, a biodegradable thermoplastic, produced in transgenic plants. Science 256:520-523

Pötter M, Madkour MH, Mayer F, Steinbüchel A (2002) Regulation of phasin expression and polyhydroxyalkanoate (PHA) granule formation in Ralstonia eutropha H16. Microbiology 148:2413-2426

Runquist D, Hahn-Hägerdal B, Bettiga M (2009) Increased expression of the oxidative pentose phosphate pathway and gluconeogenesis in anaerobically growing xylose-utilizing Saccharomyces cerevisiae. Microb Cell Fact 8:1

Sandström AG, De Las Heras AM, Portugal-Nunes D, Gorwa-Grauslund MF (2015) Engineering of Saccharomyces cerevisiae for the production of poly-3-d-hydroxybutyrate from xylose. AMB Express. doi:10.1186/ s13568-015-0100-0

Schlegel H, Gottschalk G, Von Bartha R (1961) Formation and utilization of poly-beta-hydroxybutyric acid by Knallgas bacteria (Hydrogenomonas). Nature 191:463-465

Suriyamongkol P, Weselake R, Narine S, Moloney M, Shah S (2007) Biotechnological approaches for the production of polyhydroxyalkanoates in microorganisms and plants-a review. Biotechnol Adv 25:148-175
Taherzadeh MJ, Eklund R, Gustafsson L, Niklasson C, Lidén G (1997) Characterization and fermentation of dilute-acid hydrolyzates from wood. Ind Eng Chem Res 36:4659-4665

Trotsenko YA, Belova L (2000) Biosynthesis of poly (3-Hydroxybutyrate) and poly (3-hydroxybutyrate-co-3-hydroxyvalerate) and its regulation in bacteria. Microbiology 69:635-645

Verlinden RA, Hill DJ, Kenward M, Williams CD, Radecka I (2007) Bacterial synthesis of biodegradable polyhydroxyalkanoates. J Appl Microbiol 102:1437-1449

Vijayasankaran N, Carlson R, Srienc F (2005) Synthesis of poly [(R)-3-hydroxybutyric acid) in the cytoplasm of Pichia pastoris under oxygen limitation. Biomacromolecules 6:604-611

Wang F, Lee SY (1997) Poly (3-Hydroxybutyrate) production with high productivity and high polymer content by a fed-batch culture of Alcaligenes latus under nitrogen limitation. Appl Environ Microbiol 63:3703-3706

Weusthuis RA, Lamot I, van der Oost J, Sanders JP (2011) Microbial production of bulk chemicals: development of anaerobic processes. Trends Biotechnol 29:153-158

Yalcin SK, Bozdemir MT, Ozbas ZY (2010) Citric acid production by yeasts: fermentation conditions, process optimization and strain improvement. Curr Res Technol Educ Top Appl Microbiol Microbial Biotechnol 9:1374-1382

Yoneyama F, Yamamoto M, Hashimoto W, Murata K (2015) Production of polyhydroxybutyrate and alginate from glycerol by Azotobacter vinelandii under nitrogen-free conditions. Bioengineered 6:209-217

\section{Submit your manuscript to a SpringerOpen ${ }^{\circ}$ journal and benefit from:}

- Convenient online submission

- Rigorous peer review

- Immediate publication on acceptance

- Open access: articles freely available online

- High visibility within the field

- Retaining the copyright to your article

Submit your next manuscript at $\boldsymbol{\nabla}$ springeropen.com 